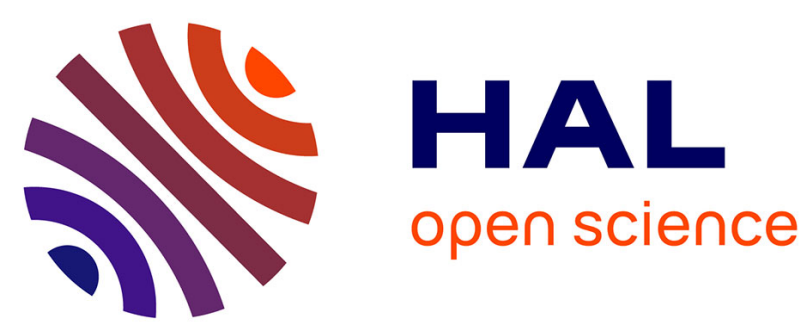

\title{
Three dimensional X-ray Diffraction Contrast Tomography Reconstruction of Polycrystalline Strontium Titanate during Sintering and Electron Backscatter Diffraction Validation
}

M. Syha, W. Rheinheimer, B. Loedermann, A. Graff, A. Trenkle, M. Baeurer, D. Weygand, Wolfgang Ludwig, P. Gumbsch

\section{To cite this version:}

M. Syha, W. Rheinheimer, B. Loedermann, A. Graff, A. Trenkle, et al.. Three dimensional X-ray Diffraction Contrast Tomography Reconstruction of Polycrystalline Strontium Titanate during Sintering and Electron Backscatter Diffraction Validation. 2nd World Congress on Integrated Computational Materials Engineering (ICME), The Minerals, Metals \& Materials Society (TMS), Jul 2013, Salt Lake City, United States. pp.259-264, 10.1007/978-3-319-48194-4_42 . hal-01706190

\author{
HAL Id: hal-01706190 \\ https://hal.science/hal-01706190
}

Submitted on 10 Feb 2018

HAL is a multi-disciplinary open access archive for the deposit and dissemination of scientific research documents, whether they are published or not. The documents may come from teaching and research institutions in France or abroad, or from public or private research centers.
L'archive ouverte pluridisciplinaire HAL, est destinée au dépôt et à la diffusion de documents scientifiques de niveau recherche, publiés ou non, émanant des établissements d'enseignement et de recherche français ou étrangers, des laboratoires publics ou privés. 


\title{
Three dimensional X-ray Diffraction Contrast Tomography Reconstruction of Polycrystalline Strontium Titanate during Sintering and Electron Backscatter Diffraction Validation
}

\author{
M. Syha ${ }^{1}$, W. Rheinheimer ${ }^{1}$, B. Loedermann ${ }^{1}$, A. Graff ${ }^{2}$, A. Trenkle ${ }^{1}$, M. Baeurer ${ }^{1}$, D. \\ Weygand $^{1}$, W. Ludwig ${ }^{3}$, P. Gumbsch ${ }^{1,2}$ \\ ${ }^{1}$ Karlsruhe Institute of Technology, IAM, Kaiserstr. 12, 76128 Karlsruhe, Germany \\ ${ }^{2}$ Fraunhofer IWM, Walter-Hülse-Str. 1, 06120 Halle, Germany \\ ${ }^{3}$ European Synchrotron Radiation Facility, 6 Rue Jules Horowitz, 38000 Grenoble, \\ France
}

Keywords: Diffraction Contrast Tomography, Sintering, EBSD, 3D Materials

Characterization, Microstructure Evolution

\begin{abstract}
The microstructural evolution of polycrystalline strontium titanate was investigated in three dimensions (3D) using X-ray diffraction contrast tomography (DCT) before and after ex-situ annealing at $1600^{\circ} \mathrm{C}$. Post-annealing, the specimen was additionally subjected to phase contrast tomography (PCT) in order to finely resolve the porosities. The resulting microstructure reconstructions were studied with special emphasis on morphology and interface orientation during microstructure evolution. Subsequently, cross-sections of the specimen were studied using electron backscatter diffraction (EBSD). Corresponding cross-sections through the 3D reconstruction were identified and the quality of the reconstruction is validated with special emphasis on the spatial resolution at the grain boundaries, the size and location of pores contained in the material and the accuracy of the orientation determination.
\end{abstract}

\section{Introduction}

Only recently, time resolved 3D characterization of polycrystalline bulk materials during microstructure evolution became feasible through non-destructive imaging techniques such as 3D X-ray diffraction microscopy $[1,2,3]$, X-ray DCT [4, 5] and differential aperture X-ray microscopy [6]. Being of invaluable use for investigations of sintering and microstructure evolution in ceramics, the DCT technique is applied here to the investigation of the annealing behavior of strontium titanate. Since the applied procedure for the analysis of diffraction data makes simplifying assumptions concerning the optics and the stress/strain state of the material [5] one focus of the present work lies on the validation of the resulting microstructure reconstructions by means of EBSD measurements taken in a subvolume of special interest. This region contains several big grains exhibiting a cube like shape and a preferred $<100>$ interface orientation [7]. Moreover, statistics on orientation distributions and morphology evolution are investigated in the context of interface property anisotropies. 


\section{Methods}

\section{Sample Preparation}

The polycrystalline DCT specimen was prepared from bulk strontium titanate made from $\mathrm{SrTiO}_{3}$ powders processed from $\mathrm{SrCO}_{3}$ and $\mathrm{TiO}_{2}$ (both 99.9+\%, Sigma Aldrich Chemie, Taufkirchen, Germany). The raw material was processed by the mixed oxide route using a molar $\mathrm{Sr} / \mathrm{Ti}$ ratio of 0.996 . After milling, calcining and isostatical pressing, the green bodies were sintered for $1 \mathrm{~h}$ at $1600^{\circ} \mathrm{C}$ in oxygen atmosphere yielding a material with an average grain radius of $14.1 \pm 1.5 \mu \mathrm{m}$. The DCT specimen was fabricated manually using a turning lathe and abrasive paper. The final sample dimensions are cylindric with $\sim 280 \mu \mathrm{m}$ diameter and a height of $\sim 350 \mu \mathrm{m}$. In between the two DCT scans, the specimen was annealed for $1 \mathrm{~h}$ at $1600^{\circ} \mathrm{C}$ in air. Detailed information on fabrication and annealing of the specimens are provided elsewhere $[8,9,7]$.

\section{Diffraction Contrast Tomography}

DCT scans of the specimen before and after heating were performed using monochromatic synchrotron X-rays, set-up and technical details as described in [4, 7]. Diffraction as well as absorption information was acquired in $360^{\circ}$ scans with an angular stepping of $0.05^{\circ}$. Using the full $360^{\circ}$ rotation allows the exploitation of pairs of diffraction spots separated by $180^{\circ}$ (Friedel pairs), which in turn allow to extract position and crystallographic orientation for each grain with high accuracy. Applying algebraic reconstruction to sets of Friedel pairs identified for a particular grain yields a 3D voxelated reconstruction of its shape. Placing the grains at their correct position inside the sample volume yields the microstructure reconstruction, which is complemented by a set of Rodriguez vectors, defining the crystallographic orientations of the individual grains. A detailed description of the data analysis procedure can be found in $[5,7]$. For the post-annealing stage, these informations were complemented by PCT [10] data. The acquisition at a larger sample-detector distance allows a free space propagation leading to edge enhancement (Fresnel diffraction) which increases the visibility of small porosities. All synchrotron experiments were performed at beamline ID11 of the European Synchrotron Radiation Facility (ESRF).

\section{Electron Backscatter Diffraction}

For the EBSD measurements, the specimen was sectioned perpendicular to the cylinder axis starting from the cylinder top. Therefore, the specimen and four silicon wafer pieces were embedded in epoxin resin. The silicon ensures the electrical conductivity and the mechanical stability of the embedding and allows depth control during abrasion and alignment. Since automated focused ion beam ablation is not feasible considering the large surface area and the hardness of the material, the sectioning was done by mechanical grinding and polishing. Prior to each EBSD scan, the specimen was polished by Argon ion beam and covered with a thin carbon film. Mounted with $70^{\circ}$ tilt to the EBSD detector in a working distance of $11 \mathrm{~mm}$, the whole cross section was scanned in a hexagonal grid with $1 \mu \mathrm{m}$ step size. Diffraction patterns were indexed using the cubic Perovskite structure with $3.905 \AA$ lattice constant and spacegroup 221. Neighboring pixels with 

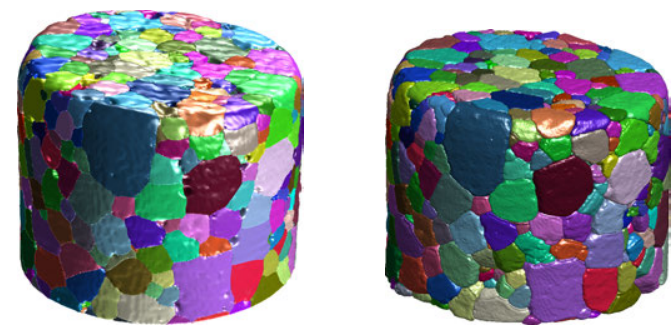

Figure 1: 3D reconstructions before (a) and after annealing (b).

orientation deviations below $3^{\circ}$ were grouped as grains. A scanning electron microscope (Zeiss; Supra $55 \mathrm{VP}$ ) equipped with an EBSD system (EDAX TSL) was used at $15 \mathrm{kV}$. Resulting images were used to generate grain boundary networks to be matched with corresponding cross-sections of the reconstructed microstructure. A detailed description of the identification of corresponding cross-sections can be found in [11].

\section{Results}

3D microstructure reconstructions of the specimen at both stages in microstructure evolution are presented in figure 1. The overall shape is identical and surface grains can easily be reidentified. The growth of the outer grains is reduced due to surface grooving effects, hindering the free motion of the grain boundaries. In order to determine the growth dynamics and porosity evolution, both reconstructions were aligned and identical subvolumes identified. The in-depth comparison of $25 \mu \mathrm{m}$ thick layer of the reconstructed volumes at both stages shown in figure 2(a) reveals the grain coarsening process. The image shows an overlay of several sections of the specimen prior to (magenta) and post (green) annealing, reflecting microstructural changes in a quasi 3D view. Figure 2(b) shows an overlay of the pores at both stages. Although pore clusters that stayed can be reidentified in the second stage, a decrease of the volume fraction of porosity was observed. From the tomography measurement the total pore volume for both stages is calculated. The volume fraction of porosity decreased from $2.6 \mathrm{vol}-\%$ in the initial stage to 1.2 vol- $\%$ in the post-annealing stage. During annealing, the number of grains changed from 849 to 797 resulting in an average volume growth of $5 \%$ per grain, under consideration of the varying porosity. Overall, the grains grow from an average grain radius of $14.7 \pm 2 \mu \mathrm{m}$ before annealing to $15.0 \pm 2 \mu \mathrm{m}$ after annealing.

Distributions of the local interface orientations in the pre- and post-annealed stage are given in multiples of the random distribution in figure 3. Orientation information has been extracted from Laplace smoothend surface tessellations of the grains that conserve the physically relevant microstructure elements. The distributions show a preference for certain interface orientations, reflected in a cumulation of $15 \%$ and $20 \%$, respectively with respect to the random distribution. An overlay of grain boundary networks as obtained by EBSD and DCT for one corresponding cross-section is given in figure 4, alongside with an euclidean distance map [12] for these corresponding cross-sections pro- 


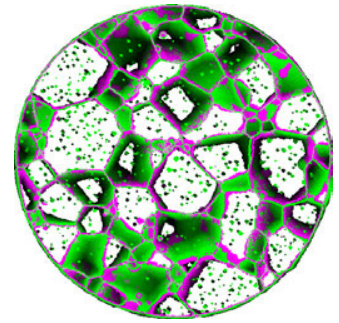

(a)

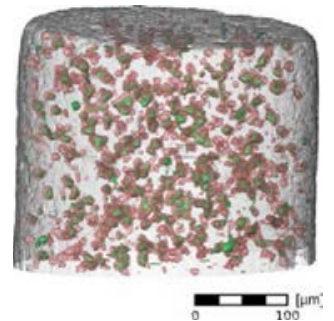

(b)

Figure 2: (a) $25 \mu \mathrm{m}$ thick layer of the reconstructed structure before (magenta) and after (green) annealing, (b) collective pore ensembles before (red) and after (green) annealing.
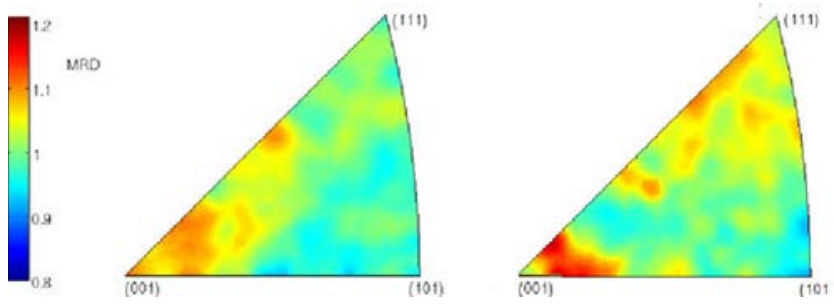

Figure 3: Distribution of local interface orientations for all bulk grains in the (a) preand (b) post-annealing state given in multiples of the random distribution.

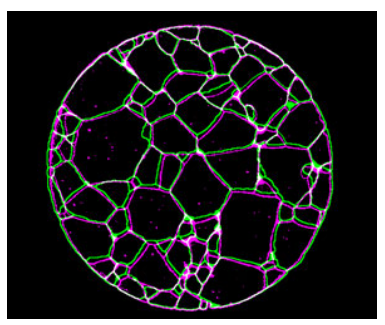

(a)

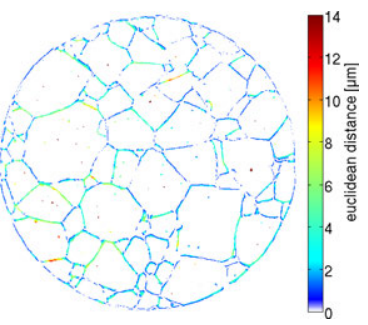

(b)

Figure 4: (a) Overlay of grain boundary networks obtained by DCT (green) and EBSD (magenta), (b) DCT network colored according to euclidean distance from EBSD network. 
jected on the DCT network. Clearly, the EBSD network shows a higher number of small intragranular pores. Apart from the disagreements resulting from these pores, deviations are mainly observed along the grain boundaries and not on triple points. The average euclidean distance between the corresponding grain boundary networks was found to be $2.26 \mu \mathrm{m}$ for the cross-section shown in 4 . The number of grains found in both networks is fairly identical, as is the average grain size [11].

\section{Discussion}

The presented results prove the feasibility of $4 \mathrm{D}$ microstructure characterization in perovskites using X-ray DCT. High resolution grain orientations allow the reidentification of crystallites after heating so that the two microstructure reconstructions could be aligned for further investigations of volume, orientation and topology characteristics at the different stages. The reconstructed microstructures exhibit moderate grain coarsening and densification, the latter being most likely underestimated due to the fact that the porosity was measured with higher accuracy for the post annealing stage. The error of $2 \mu \mathrm{m}$ in the average grain size is the estimated error introduced by volumetric measurement of the average grain size in the finished reconstruction. However, additional uncertainty might be introduced by the reconstruction algorithm, which contains a dilation step for filling gaps in the initial voxel information [5]. Considering the resolution limit for small grains, which is about 300 voxels and using a greedy algorithm to fill the gaps in the undilated structure with smaller grains yields a more robust error estimate: Allowing for grains down to 200 voxels volume yields a change of $5.5 \mu \mathrm{m}$ in mean radius. However, the good agreement between EBSD and DCT characterization gives reason to assume the error introduced by the dilation procedure to be much smaller. Reported deviations between EBSD and DCT might occur due to uneven cutting plane resulting from the manual preparation of the cross sections for EBSD measurements.

Interface orientation distributions reveal a preference for orientations lying within a $5^{\circ}$ range of the $\langle 100\rangle$ orientation, which is in excellent agreement with the results from orientation imaging microscopy [13]. This orientation is the low energy orientation in the $\mathrm{SrTiO}_{3}$ system [14]. The accuracy of the orientation determintaion is affected by the smoothing step. That said the momentary achievable accuracy is not yet sufficient to identify general anisotropies in grain boundary properties except for pronounced cases of faceting. DCT with higher resolution and/or a refined reconstruction technique might improve the spatial resolution at the grain boundaries. A significantly improved resolution of the porosity $(30-50 \mathrm{~nm})$ might be achieved by the application of optimized optical instruments in combination with Zoom-tomography [15].

\section{References}

[1] E. Lauridsen et al. "Growth kinetics of individual cube grains as studied by the 3D X-ray diffraction microscope". Recrystallizationd and Grain Growth. Proceedings, 1, (2001), 589-594.

[2] H. Poulsen. Three-dimensional X-Ray diffraction microscopy. Mapping polycrystals and their dynamics (Springer, 2004). 
[3] R. Suter et al. "Forward modeling method for microstructure reconstruction using x-ray diffraction microscopy: Single-crystal verification". Review of Scientific Instruments, 77, (2006), 123905.

[4] G. Johnson et al. "X-ray diffraction contrast tomography: a novel technique for three-dimensional grain mapping of polycrystals. I. The combined case". Journal of Applied Crystallography, 41, (2008), 310-318.

[5] W. Ludwig et al. "Three-dimensional grain mapping by x-ray diffraction contrast tomography and the use of Friedel pairs in diffraction data analysis". Review of Scientific Instruments, 033905.

[6] B. Larson et al. "Three-dimensional X-ray structural microscopy with submicrometre resolution". Nature, 415, (2002), 887-890.

[7] M. Syha et al. "Three-dimensional grain structure of sintered bulk strontium titanate from X-ray diffraction contrast tomography". Scripta Materialia, 66, (2012), $1-4$.

[8] M. Bäurer et al. "Grain growth anomaly in strontium titanate". Scripta Materialia, 61 (6), (2009), $584-587$.

[9] M. Bäurer, H. Kungl, and M. Hoffmann. "Influence of Sr/Ti Soiciometry on the Densification Behaviour of Strontium Titanate". Journal of the American Ceramical Society, 92 (3), (2009), 601-606.

[10] P. Cloetens et al. "Observation of microstructure and damage in materials by phase sensitive radiography and tomography". Journal of Applied Physics, 81, (1997), 5878 .

[11] M. Syha et al. "Validation of 3D diffraction contrast tomography reconstructions by means of EBSD characterization". Journal of Applied Crystallography, subm.

[12] P. Danielsson. "Euclidean Distance Mapping". Computer Graphics and Image Processing, 14, (1980), 227-248.

[13] D. Saylor et al. "Distribution of Grain Boundaries in SrTiO3 as a Function of Five Macroscopic Parameters". Journal of the American Ceramical Society, 84 (4), (2004), 670-676.

[14] T. Sano, D. Saylor, and G. Rohrer. "Surface Energy Anisotropy of SrTiO3 at 1400 ${ }^{\circ} \mathrm{C}$ in Air". Journal of the American Ceramical Society, 86 (11), (2003), 1933-1939.

[15] R. Mokso et al. "Nanoscale zoom tomography with hard X-rays using KirkpatrickBaez optics". Applied Physics Letters, 90, (2007), 144107. 